\title{
Caracterización de escorias metalúrgicas procedentes de yacimientos arqueológicos de Navarra (Siglos II a.C.- IV d.C.) $)^{(\bullet)}$
}

\author{
L. Ros-Latienda* y J. Fernández-Carrasquilla*
}

\begin{abstract}
Resumen
Desde la Sección de Arqueología del Gobierno de Navarra llegó la propuesta de caracterizar una serie de piezas comprendidas entre el siglo II a.C. y el siglo IV d.C. procedentes de yacimientos arqueológicos de esta provincia con objeto de obtener datos relacionados con la tecnología que las produjo y poder comparar el grado de desarrollo técnico de diferentes épocas y lugares. Interesaba el estudio de estas piezas desde el punto de vista metalúrgico, habiendo entre ellas mayoritariamente escorias metalúrgicas, restos de piezas fabricadas y restos minerales utilizados en la obtención de diversos metales. Además de proceder a su análisis químico mediante técnica de plasma acoplado inductivamente (ICP), se estudiaron seis muestras mediante microscopía óptica, electrónica de barrido y microanálisis por energía dispersiva de rayos X (EDAX) que reveló la composición de zonas amplias de las escorias y finalmente se completó el estudio determinando las fases presentes mediante difracción de rayos X.
\end{abstract}

Palabras clave

Arqueometalurgia; Yacimientos arqueológicos; Hierro; Escorias metalúrgicas;Caracterización; Siglo II a.C. hasta siglo IV d.C.

\section{Characterization of metallurgical slags from archaeological sites in Navarre $\left(2^{\text {nd }}\right.$ century B.C. through $4^{\text {th }}$ century A.D.)}

Abstract

Keywords

\begin{abstract}
The Archaeology Section of the Government of Navarre put forward a proposal to undertake research in the characterization of a set of pieces from $2^{\text {nd }}$ century B.C. to $4^{\text {th }}$ century A.D. retrieved from excavations in different archaeological sites in this province, to obtain data related to the manufacturing process of the investigated artefacts and to compare the level of technical skills in different times and places, so as to achieve a better understanding of technological abilities and material culture. The finds discussed in this paper are of interest from an archaeometallurgical point of view, and they consist mainly of metallurgical slags, remains of manufactured pieces, and minerals residues used to obtain various metals. In addition to carrying out their chemical analyses by inductively coupled plasma (ICP), the study of six samples was performed by optical and scanning electron microscopy and energy-dispersive X-ray microanalysis (EDX) which revealed the composition of large areas of the slags, and the study was finally completed by the determination of the phases present by $\mathrm{X}$ rays diffraction.
\end{abstract}

Archaeometallurgy; Archaeological sites; Iron; Metallurgical slags; Characterization: $2^{\text {nd }}$ century B.C. through $4^{\text {th }}$ century A.D.

\section{INTRODUCCIÓN}

El objetivo del trabajo es la caracterización de piezas comprendidas entre el siglo II a.C. y el siglo IV d.C., proporcionadas por la Sección de Arqueología del Gobierno de Navarra procedentes de yacimientos arqueológicos de esta provincia, con objeto de obtener datos relacionados con la tecnología que las produjo y poder comparar el grado de desarrollo técnico de diferentes épocas y lugares.
En relación con el estudio de los diversos tipos de hornos metalúrgicos, remitimos al lector a la consulta de los trabajos de diversos autores que indicamos en las referencias bibliográficas como Tylecote, Craddock, Gómez-Ramos y Rovira ${ }^{[1-4]}$ entre otros.

Las piezas objeto de nuestro estudio podían formar parte de una larga lista como restos de revestimientos, de escorias, de minerales, de objetos manufacturados, de restos de hornos y de subproductos metalúrgicos.

(•) Trabajo recibido el día 10 de enero de 2013 y aceptado en su forma final el día 25 de julio de 2013

* Dpto. de Ingeniería Mecánica, Energética y de Materiales, Universidad Pública de Navarra, C/ Arrosadía s/n, 31006, Pamplona, E-mail: ferca@unavarra.es 
Ofrecemos a continuación un breve resumen de sus características.

Objetos fabricados: en su estudio, la tesis de Gómez-Ramos ${ }^{[5]}$ aporta una clasificación que incluye las características para identificar las piezas a estudiar.

Revestimientos: según Rothenberg et al. ${ }^{[6]}$, las piedras y adobes que constituían el horno estaban revestidos de arcilla, material refractario que conserva en la zona en contacto con la carga coloraciones ocasionadas por el fuego, adherencias de escorias o una capa vidriada por la naturaleza alcalina de las cenizas incandescentes. El análisis químico y estructural permite determinar las temperaturas con las que se trabajaba.

Según el grado de vitrificación se puede llegar a calcular la duración del proceso de reducción, así como la temperatura alcanzada a partir de las transformaciones sufridas por los compuestos arcillosos. Hay que recordar que el vidriado se produce por encima de los $1.200^{\circ} \mathrm{C}$.

Crisoles: son de arcilla refractaria o piedra (esteatita). Para evitar su rotura durante el calentamiento, las paredes eran más gruesas y tendían a utilizarse tierras pobres en silicato de aluminio y más ricas en cuarzo. Pueden parecer de tamaño pequeño pero no lo son tanto si se tiene en cuenta la densidad del metal.

Moldes: deben existir desde el origen de la metalurgia por lo que pueden ser de piedra, arcilla o metal. Las formas básicas serían: abiertos (univalvos) y los procedentes del moldeo a la cera perdida (cerrados bivalvos o polivalvos). El precalentamiento podría mejorar el desmoldeo al formarse una pequeña película de óxido de lo cual puede también haber huella.

\section{Subproductos:}

a) Minerales parcialmente reducidos. Es normal encontrar en los yacimientos este tipo de producto consecuencia de una reducción incompleta, si bien para su reconocimiento habría que buscar huellas de calentamiento, como un redondeo de la superficie y estructuras internas a base de óxidos alrededor de un núcleo de mineral original.

b) Lingotes de metal. Hay pocos vestigios de aleaciones de cobre, que se pueden confundir con materia prima. No se encuentran restos de lingotes de hierro debido a la imposibilidad de obtener hierro fundido en los hornos antiguos; en este caso las formas se obtenían a partir del trabajo de martillado del hierro bruto.

c) Escorias de producción metalúrgica. Las escorias metalúrgicas que interesan en este trabajo pueden ser confundidas con otro tipo de material formado por la acción del fuego. Hauptmann ${ }^{[7]}$ presenta la siguiente relación:
Material geológico (escorias naturales): lava, piedra pómez.

Escorias de origen doméstico y de destrucción: ladrillos vitrificados, cenizas.

Escorias no metalúrgicas: manufactura de ladrillos, cal, cerámica, vidrio.

Escorias metalúrgicas:

- fundición de minerales no férreos

- fundición de metales no ferrosos

- hornos bajos de reducción para la producción de hierro

- forja de hierro

Obviamente, el análisis de laboratorio es imprescindible para diferenciar unas de otras. Con el fin de profundizar más en los aspectos tecnológicos del proceso siderúrgico, hemos llevado a cabo también un estudio de las fases mineralógicas presentes en la escoria por técnicas de Difracción de Rayos X (DRX).

\section{ANÁLISIS DE ESCORIAS METALÚRGICAS}

Una vez establecido que el material del que se dispone es una escoria metalúrgica, se continúa con su análisis para determinar su origen. Su composición proporciona algunos datos acerca de los materiales, técnicas y temperaturas con los que se trabajó. Para ello es preciso saber cómo se forma una escoria tanto en lo que atañe a su composición como a la temperatura del horno.

La ayuda del microscopio electrónico de barrido (MEB), muestra el aspecto característico de determinadas zonas de la escoria; así la morfología dendrítica revela que el material realmente ha evolucionado desde un estado de completa fusión. El microanálisis por energía dispersiva de rayos X (EDAX) revela la composición de zonas amplias de la escoria.

En referencias bibliográfica citadas por Setién y Diez-Aja ${ }^{[8]}$ se encuentran resultados de análisis de escorias de hierro de distintas épocas, que abarcan desde tiempos de los romanos hasta nuestros días siendo preciso hacer notar la enorme variabilidad de resultados que se registran de unas muestras de escoria a otras, aun cuando su procedencia sea la misma.

Esta variabilidad característica refleja que los procesos de reducción de mineral aplicados en el horno eran imperfectos y no estaban totalmente bajo el control del fundidor, lo cual implicaría que las condiciones reductoras y el rendimiento en el interior del horno fueran irregulares. 


\subsection{Formación de escorias en el proceso de reducción. Composición}

La formación de escoria empieza desde el mismo momento de la obtención del metal por reducción de su mineral. Es de señalar que en el caso del hierro éste permanecería en estado sólido dado que las bajas temperaturas de trabajo de un horno antiguo no permiten obtenerlo en estado líquido.

El contenido en hierro se muestra como característica que permite distinguir las escorias de fundición de otros materiales, pero a su vez, el elevado contenido de silicatos de hierro de todas las escorias, ya sean de producción de cobre como de hierro, lleva a una clasificación incorrecta.

Si la ganga es silicatada, el fundente será óxido (generalmente de hierro, aunque también pueden ser de calcio y manganeso); si es calcárea se añade sílice (arena, cuarzo machacado). Las escorias de obtención de metal producidas en la antigüedad son básicamente silicatos de hierro cristalizados (fayalitas), lo que en el caso del hierro supone sacrificar parte del mismo para la formación de aquéllas.

Aunque la composición química puede variar según el mineral, los fundentes y las temperaturas de trabajo, los componentes más importantes de las escorias son silicatos, óxidos y metales. Entre sus conclusiones Gómez Ramos ${ }^{[5]}$ destaca el uso inadecuado de arena cuarcítica como fundente para la obtención de bronce en lugar de utilizar óxidos de hierro.

Si bien la formación de fayalita, cuya temperatura de fusión entre $1.000{ }^{\circ} \mathrm{C}$ y $1.100{ }^{\circ} \mathrm{C}$ era el objetivo básico, la presencia de $\mathrm{FeO}$ en forma de wustita y de $\mathrm{Fe}_{3} \mathrm{O}_{4}$ o magnetita es habitual en las escorias antiguas e indicaría en el primer caso, pobre aporte de sílice; el $\mathrm{FeO}$ no podría formar fayalita y quedaría en forma de wustita. La presencia del segundo indicaría malas condiciones de reducción o atmósfera poco oxidante. Asimismo, un exceso de sílice originaría igualmente una escoria poco fluida, ya que si no hay suficiente $\mathrm{FeO}$ lo que se forman son pyroxenos y clinopyroxenos, en los que el hierro se reemplaza por calcio o magnesio, lo cual es propio de metalurgias primitivas como podrían ser las del cobre. En este sentido, el trabajo de Salvador Rovira ${ }^{[4]}$ es muy esclarecedor.

La presencia de una cantidad excesiva de metal en la escoria será el indicio de una escorificación deficiente o de una temperatura en el horno por debajo de la necesaria para una buena fluidificación de las escorias.

El resto de los componentes se encontrarían formando una matriz silicatada vítrea de composición variable. Otros elementos constituyentes procederían del revestimiento, de la ganga del propio fundente e incluso del mineral que aporte el combustible, lo que a su vez da lugar a cenizas fundamentalmente alcalinas.

El trabajo de Andreas Kronz ${ }^{[9]}$, es especialmente ilustrativo de las distintas composiciones de las escorias siendo todas ellas llamadas "escorias fayalíticas". Su trabajo consiste en comparar la producción de hierro antiguo (siglos $\mathrm{V}$ al IV a.C.) y medieval (siglos VII al XIII d.C.) de sendas zonas de Alemania. De acuerdo con los resultados de sus análisis y en función del contenido de $(\mathrm{FeO}+\mathrm{MnO})$ agrupa las escorias de producción antigua en tres tipos: unas, de $77 \%$ en peso, en las que dominaría la wustita frente a la fayalita $\left(\mathrm{Fe}_{2} \mathrm{SiO}_{4}\right)$, otras con un $60 \%$ en las que la wustita decrece y la fayalita aumenta y unas terceras con un $30 \%$ que sólo se encuentran entre las antiguas en las que la fayalita juega un papel menor y es reemplazada por hedenbergita clinopyroxeno $\left(\mathrm{CaFeSi}_{2} \mathrm{O}_{6}\right)$. Aunque no presenta datos, hace la observación de que en el caso de producción medieval, el valor promedio se situaría en torno a las del $60 \%$ que, por otra parte, es el valor ideal.

El mismo trabajo refiere cómo el total de álcali y la relación álcali- $\mathrm{Al}_{2} \mathrm{O}_{3}$ es superior en los ejemplos prerromanos, lo que lleva a la conclusión de que hay gran cantidad de ceniza y por lo tanto elevada relación combustible/escoria. Al fósforo lo hace provenir del mineral goethita/limonita que se usaría en los antiguos hornos en contraposición a los medievales que usarían hematites que no contiene fósforo.

El artículo de A. Kronz ${ }^{[9]}$ presenta un hipotético "balance de masa" de las reacciones que intervienen en los procesos llegando a la conclusión de que la escoria con 30\% en peso de $\mathrm{FeO}$ sólo se puede formar en el trabajo de forja, pues requiere el consumo de hierro sin producirlo. Como dato interesante señalaremos que de ese "balance de masa" deduce que se hace innecesaria la adición específica de fundentes y que éstos provendrían del revestimiento del horno y de las cenizas del combustible.

\subsection{Temperatura}

La wustita y la magnetita se pueden presentar en forma de cristales o en dendritas. Los óxidos ferromagnéticos funden a temperaturas superiores a los $1.200^{\circ} \mathrm{C}$, por lo que la presencia de dendritas indicaría que se han alcanzado esas temperaturas aunque no pudieran mantenerse estables. La "prueba" de haber alcanzado estas temperaturas también las podemos encontrar en la presencia de granos de sílice o cuarzo sin fundir o vidriados superficialmente detectados en las estructuras, ya que requieren temperaturas del orden de $1.700^{\circ} \mathrm{C}$ muy superiores a las de los óxidos. 
Por último, cabe establecer como herramienta para tratar la composición en función de la temperatura de solidificación los diagramas de fases ternarios, en los que se sitúan la composición de los tres principales componentes de las escorias y la temperatura.

En el caso de escorias de hierro, Gómez- Ramos ${ }^{[5]}$ hace referencia a Bachman ${ }^{[10]}$ que como principal especialista en el tema, hace hincapié en el estudio de diagramas de óxido de hierro, sílice y óxidos silicatados de calcio.

Las altas temperaturas de fundición de cada especie por separado, impedirían la obtención de hierro, pero las combinaciones, por ejemplo $62-76 \%$ de óxido de hierro, en torno al $40 \%$ de sílice y bajo contenido de anortita (feldespato), permiten formar fayalita en torno a $1.100^{\circ} \mathrm{C}-1.200^{\circ} \mathrm{C}$ lo que ya es asequible a un horno antiguo. Si el contenido de óxido de hierro es superior al necesario para formar fayalita el resto quedará en forma de wustita y la encontraremos en forma de nódulos o esqueletos dendríticos según la temperatura. $\mathrm{Si}$ hay más sílice se formará tridimita o cristobalita, pero a temperaturas superiores a los $1.200^{\circ} \mathrm{C}$. Si lo que aumenta es el contenido de anortita (feldespato), se formará hercinita $\left(\mathrm{Fe}^{++} \mathrm{Al}_{2} \mathrm{O}_{4}\right)$ a $\mathrm{T}>1.300^{\circ} \mathrm{C}$ y anortita a $\mathrm{T}>1.400^{\circ} \mathrm{C}$. Como ya se ha dicho, la mayoría de las antiguas escorias son fayaliticas.

\subsection{Formación de escorias de herrería}

El hierro se obtenía sólido en forma de esponja por aglutinación de pequeñas gotitas, por lo que debía ser sometido al proceso de forja para fabricar el utensilio. Este proceso debía hacerse a base de martillado, facilitándolo previo calentamiento del metal, lo que origina otro tipo de escoria del óxido metálico que se va formando en el proceso de calentamiento del metal en atmósfera libre. Este óxido formaría una costra que se desprendería cayendo al fondo del horno donde se fundiría con otros componentes, si bien también podía ser básicamente $\mathrm{FeO}$; en cualquier caso, cada una correspondería al trabajo de un día o de una sesión, entendiendo por tal el encendido-apagado del horno.

Los procesos de forja, por ser más variados son difíciles de describir y por lo tanto las investigaciones arqueológicas en este campo no están tan desarrolladas, de ahí el interés del trabajo publicado por Serneels y Perret ${ }^{[11]}$ en el que explican la presencia de sílice en la escoria a causa de la adición de arena durante el calentamiento. La arena haría de envoltura protectora de la pieza que evitaría su oxidación durante el calentamiento, particularmente en piezas grandes. En su artículo describen la formación de lo que para ellos es la "típica" escoria de forma plano-convexa PCB (plano-convex bottom) y encuadrándola dentro de ocho tipos en función de la proporción de los tres materiales más importantes que las conforman. El primero estaría dominado por fayalita con cantidades variables de óxido de hierro (preferentemente wustita) y pequeñas cantidades de vidrio intersticial conocido por SGD (scorie grise dense o dense grey slag) y sería similar al formado en los procesos de fundición. El segundo tipo sería rico en sílice y otros materiales comúnmente encontrados en las rocas SAS (scorie argilo-sableuse o sandy-clayey slag). El tercer tipo sería rico en hierro que podría presentarse como tal metal o formando óxido e hidróxidos y que frecuentemente presenta inclusiones de carbón y podría ser magnética SFR (scorie ferreuse rouillée o iron-rich rusty slag).

Del estudio de distintos trabajos y a modo de conclusión, podemos definir los elementos que debemos investigar en nuestras propias escorias y cuáles son las técnicas más adecuadas para ese fin.

\section{MATERIALES, METODOLOGÍA Y TÉCNICAS EXPERIMENTALES}

\subsection{Materiales}

Como recomendación para la periodización de la Edad del Hierro en el territorio de Navarra, es importante considerar el trabajo de J. Armendáriz ${ }^{[12]}$ ya que no sería correcto usar el término "celtiberización" puesto que la cultura ibérica se delimita fundamentalmente en la franja mediterránea de la Península Ibérica. Para el inicio de la Edad del Hierro en Navarra (Primera Edad del Hierro o Hierro Antiguo) según Armendáriz ${ }^{[12]}$ : "Hierro Antiguo nos referiremos al desarrollo que esta cultura tiene entre finales del siglo VIII a.C. y los últimos años del VI".

Así, desde el punto de vista arqueológico, las piezas objeto de estudio pertenecen a un período de tiempo que transcurre desde, aproximadamente, el siglo II a.C. (Segunda Edad del Hierro o Edad del Hierro Final según Armendáriz ${ }^{[12]}$ ) hasta época romana en el siglo IV d.C., por ello es de suponer que en tan dilatado espacio de tiempo la tecnología del hierro sufrió una considerable evolución.

Las piezas objeto de estudio, junto con su yacimiento, han sido referenciadas como se indica a continuación:

Pieza n 1: Escorias "San Saturnino-Artajona (área 2-3 U. E.), 7 julio 2003".

Pieza $n^{\circ}$ 2: Escorias "Andelos" 85-86 Sc. Andueza Cata 11c.

Pieza n ${ }^{\circ}$ 3: "Sartaguda Yac. 15 La Cerradilla Excavaciones de 1961". 
Pieza n ${ }^{\circ}$ 4: Escorias "Areso escorias" Tres piezas. Pieza n ${ }^{\circ}$ 5: "Arellano, Alto Cárcel".

Pieza $n^{\circ}$ 6: "Arellano, 2 hierros".

En la figura1 se muestran las fotografías de las piezas de los seis yacimientos junto con su descripción.

\subsection{Metodología y técnicas experimentales}

La caracterización de las muestras, siguiendo el planteamiento de Rosado y García ${ }^{[13]}$ se realizó aplicando ensayos clásicos tales como la inspección visual y metalográfica hasta análisis mediante espectroscopía ICP, MEB y DRX.

Se siguieron las siguientes etapas:

1) Recepción de la pieza: toma de fotografías antes y después de limpiarla, recogida de datos arqueológicos, yacimiento, entorno, etc. así como masa, densidad, magnetismo residual, dimensiones y cuantas características morfológicas se consideraron significativas.

2) Obtención de una muestra en polvo de cada una de las piezas, procediendo a su trituración y haciendo pasar el polvo por un tamiz de $0,18 \mathrm{~mm}$ para facilitar su fusión a $900^{\circ} \mathrm{C}$ en crisol de platino de $0,2 \mathrm{~g}$ de muestra mezclada con carbonato y tetraborato sódico y posterior disolución de las sales en ácido clorhídrico. La determinación de sodio y potasio tuvo lugar sobre otra muestra de $0,2 \mathrm{~g}$ atacada con mezcla de ácidos fluorhídrico, nítrico y clorhídrico.
Finalmente se procedió a realizar el análisis químico de los elementos habituales en esta clase de muestras (hierro, silicio, calcio, sodio, aluminio, potasio, cobre y fósforo) mediante un espectrómetro de emisión por plasma acoplado inductivamente ARL Mod. 3410. Las curvas de calibración se prepararon a partir de soluciones de 10 y 100 ppm obtenidas a partir de disoluciones de $1.000 \mathrm{ppm}$ de la marca Alpha (Tabla I).

3) Después del corte y pulido de las muestras procedentes de las piezas tanto atacadas con reactivos ácidos como sin atacar, se procede a su observación mediante microscopía óptica y electrónica de barrido y a la toma de las correspondientes microfotografías (Fig. 2). La observación de fases mediante un microscopio óptico invertido Olympus Mod. PME 3-313 UN se realizó previa preparación siguiendo la metodología convencional de empastillado, lijado y pulido.

4) Identificación de las fases e incrustaciones presentes mediante análisis por difracción de rayos $\mathrm{X}$ de polvo obtenido de diferentes partes de la pieza (Fig. 3).

Las medidas se realizaron a temperatura ambiente usando un "D-Max Rigaku" provisto de un ánodo rotante. El difractómetro funciona a $40 \mathrm{Kv}$ y $80 \mathrm{~mA}$, con un ánodo de $\mathrm{Cu}$ y se utilizó un monocromador de grafito para seleccionar la radiación $\mathrm{CuK \alpha} 1,2$. Los datos se recogieron de $2 \Theta=5^{\circ}$ a $80^{\circ}$ con un paso de $0,03 \mathrm{yt}=1 \mathrm{~s} /$ paso.

Las muestras (escorias, básicamente amorfas) al ser poco cristalinas y consistir en mezclas de varias fases, muestran orientación preferencial, lo que da lugar a que los correspondientes picos de difracción sean más inten-
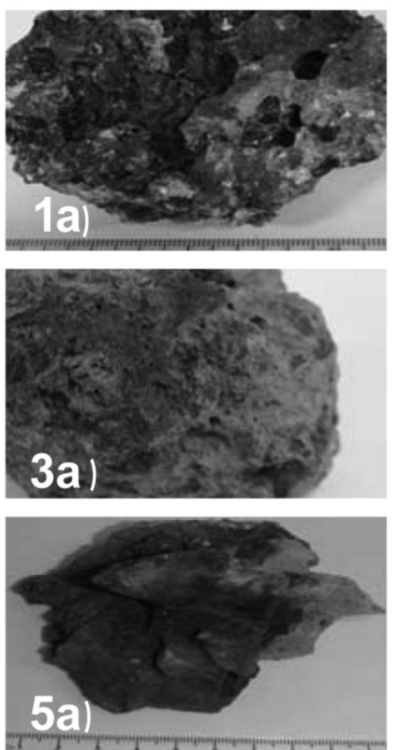
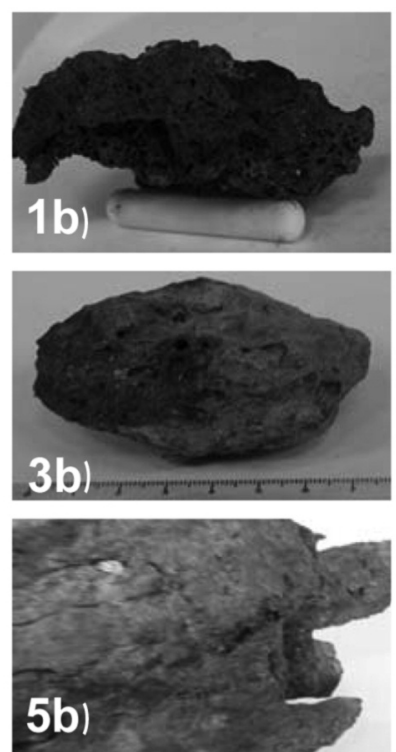
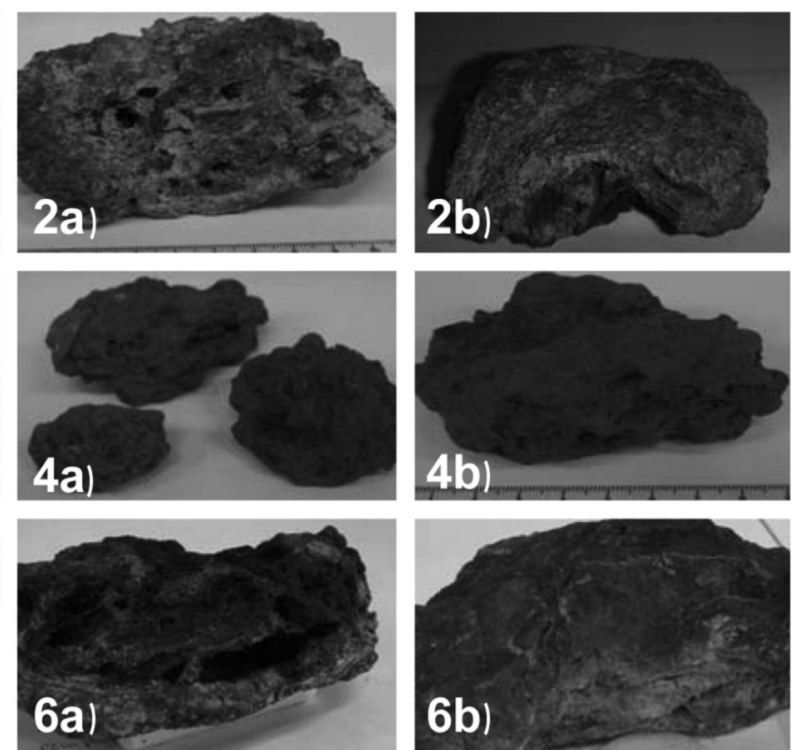

Figura 1. Fotografías de las piezas de los seis yacimientos.

Figure 1. Photographs of the pieces from the six sites. 
CARACTERIZACIÓN DE ESCORIAS METALÚRGICAS PROCEDENTES DE YACIMIENTOS ARQUEOLÓGICOS DE NAVARRA (SIGLOS II A.C.- IV D.C.) CHARACTERIZATION OF METALLURGICAL SLAGS FROM ARCHAEOLOGICAL SITES IN NAVARRE (2 ${ }^{\mathrm{ND}}$ CENTURY B.C. THROUGH $4^{\text {TH }}$ CENTURY A.D.)

Tabla I. Análisis mediante ICP de las piezas (\% en peso)

Table I. Analysis ICP of pieces (weight \%)

\begin{tabular}{|c|c|c|c|c|c|c|c|c|c|c|c|}
\hline Pieza & $\mathrm{FeO}$ & $\mathrm{SiO}_{2}$ & $\mathrm{CaO}$ & $\mathrm{BaO}$ & MnO & $\mathrm{Al}_{2} \mathrm{O}_{3}$ & MgO & $\mathrm{Na}_{2} \mathrm{O}$ & $\mathrm{K}_{2} \mathrm{O}$ & $\mathrm{Cu}_{2} \mathrm{O}$ & $\mathrm{P}_{2} \mathrm{O}_{5}$ \\
\hline No 1 & 50,0 & 18,3 & 8 & 5 & 0,010 & 2,2 & 0,75 & 0,006 & 0,36 & 0,30 & 0,32 \\
\hline 02 & 53,0 & 16,7 & 11,2 & 0,006 & 0,08 & 2,6 & 1,00 & 0,007 & 0,62 & 0,06 & 0,96 \\
\hline$N^{\circ} 3$ & 47,6 & 42,7 & 1,6 & 0,018 & 0,05 & 2,3 & 1,0 & 0,007 & 1,0 & $<0,005$ & 0,57 \\
\hline$V^{0} 4$ & 47,1 & 41,3 & 2,0 & 0,015 & 0,17 & 5,4 & 1,26 & 0,39 & 1,6 & $<0,005$ & 0,55 \\
\hline$N^{\circ} 5$ & 80 & 3,6 & 0,24 & 0,005 & 0,008 & 0,6 & 0,17 & 0,10 & 0,01 & $<0,005$ & 0,17 \\
\hline$N^{\circ} 6$ & 97,1 & 4,7 & 0,4 & 0,3 & 0,008 & 0,2 & 0,06 & 0,09 & 0,01 & $<0,005$ & 0,09 \\
\hline
\end{tabular}

sos de lo que deberían ser en realidad. Todo ello hizo que la determinación de fases resultara complicada.

Con este método sólo se pueden determinar fases que se encuentren en una proporción mayor al 1\%, por lo que no se pudieron detectar elementos tales como cobre o fósforo.

\section{RESULTADOS}

Pieza n ${ }^{\circ}$ : San Saturnino-Artajona-área 2-3 U. E. 7 julio 2003.

Información arqueológica y descripción morfológica: Fondo de horno, escorias, dentro de la iglesia de San Saturnino. En estructura romana relacionada con la época del Alto Imperio s. I-II. No presenta magnetismo residual en pieza, pero sí en polvo. Como se observa en las fotografías, tiene forma plano-convexa, con mucha porosidad y algunos alvéolos muy grandes; color negruzco y tierra pegada básicamente en la parte convexa. Analizada la tierra externa se comprobó que se trataba de carbonatos. En la cara superior aparecen algunas manchas de óxido ambiental. Otras manchas, negras, podrían indicar la presencia de carbón. Una vez limpia, su aspecto y forma no cambia apreciablemente. Masa antes de limpiarla $=181,1 \mathrm{~g}$, después $=176,9 \mathrm{~g}$.

Densidad: $2,594 \mathrm{~g} / \mathrm{cm}^{3}$ (resultado aproximado dada su porosidad).

$\mathrm{Su}$ aspecto sugiere que se trata de una escoria que conserva la forma del fondo del recipiente que la contenía ¿posible horno pequeño?, el análisis por DRX indica la presencia de carbono y azufre que no pueden
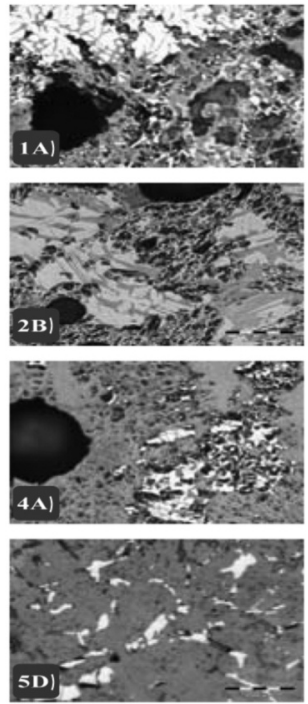
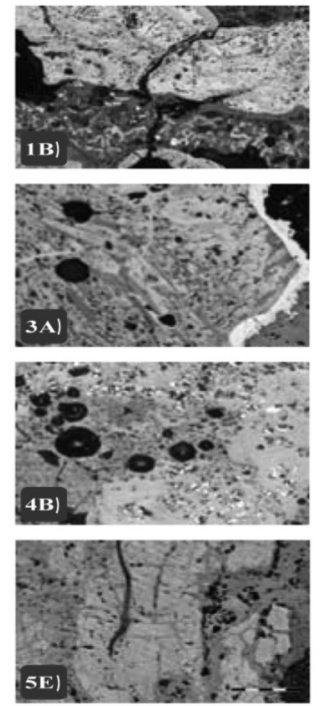
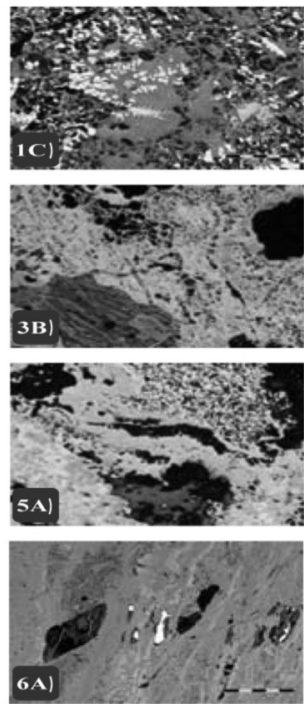
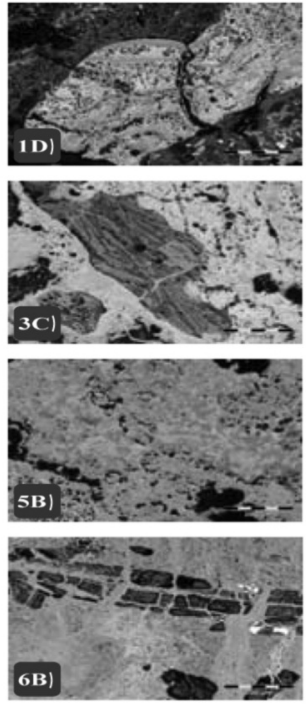
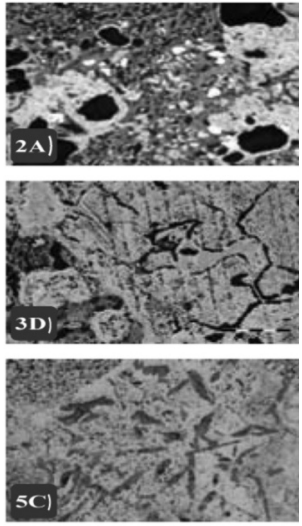

Figura 2. Microfotografías obtenidas mediante microscopía óptica (x100) de las muestras pulidas sin ataque y pulidas y atacadas.

Figure 2. Microphotographs obtained by optical microscopy (x100) of polished-unetched and polished and etched samples. 

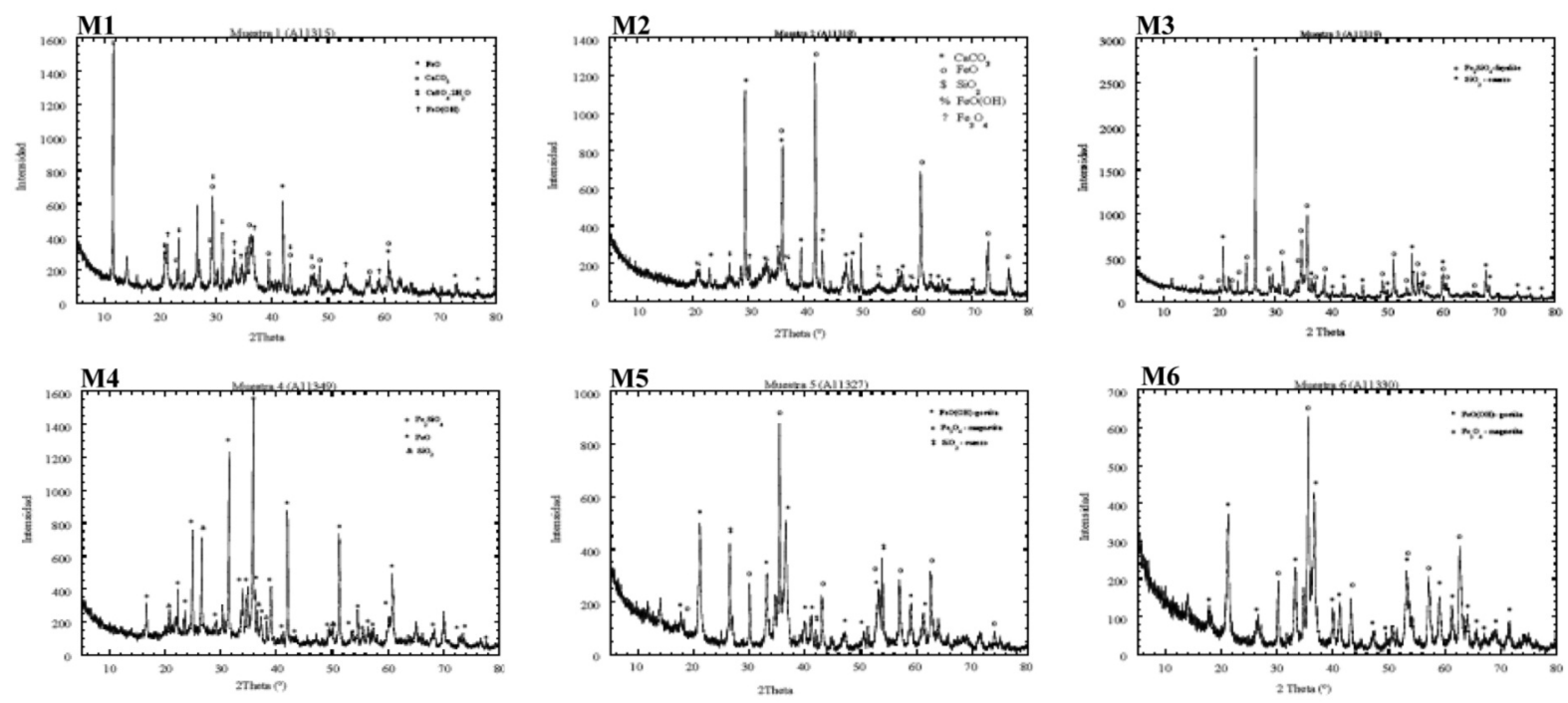

Figura 3. Difractogramas realizados sobre muestras de los seis yacimientos.

Figure 3. Diffractograms obtained from samples from the six sites.

ser cuantificados mediante ICP. La muestra presenta tres fases principales: calcita $\left(\mathrm{CaCO}_{3}\right)$, yeso $\left(\mathrm{CaSO}_{4} \cdot 2 \mathrm{H}_{2} \mathrm{O}\right)$ y wustita $(\mathrm{FeO})$. También se observan picos correspondientes a magnetita $\left(\mathrm{Fe}_{3} \mathrm{O}_{4}\right)$, goethita $(\mathrm{Fe}(\mathrm{OH}))$ y cuarzo $\left(\mathrm{SiO}_{2}\right)$. La excesiva concentración de hierro frente a silicio indica que no se formó fatalita; corroborada su ausencia mediante observación microscópíca, nos lleva a considerarla como una mala escoria de hierro (de horno de reducción). Solamente se llegó a formar escoria de tipo clinopiroxeno que incluiría el contenido relevante de carbonato de calcio. La observación por MEB revela la existencia de formaciones blancas de bordes redondeados de wustita como se podía prever por el exceso de hierro. Hay otro tipo de manchas blancas, puntuales, más brillantes, que no se han podido identificar por MEB y que podrían ser de metal retenido, lo que es normal dada la poca fluidez que debería tener la escoria. Aunque el contenido de cobre es bajo, podría tratarse de una escoria de cobre en cuyo caso la presencia de óxido de hierro indicaría una mala técnica. En una escoria de hierro no tendría por qué haber cobre, aunque sí podría darse el caso contrario. El análisis por DRX del cobre no es posible si está en cantidades bajas, así que sólo el estudio mediante MEB podría resolver la pregunta. El hierro podría provenir de los minerales de cobre: bornita $\left(\mathrm{S}_{4} \mathrm{FeCu}_{3}: 63,4 \%\right)$ y calcopirita $\left(\mathrm{S}_{4} \mathrm{FeCu}: 34,5 \%\right)$ que aunque contienen hierro no se consideran menas de ese elemento.

En la figura 1 , correspondiente al yacimiento $\mathrm{n}^{\circ} 1$ "Escorias San Saturnino-Artajona", en la fotografía 1 a) se observan los alvéolos y el aspecto poco homogéneo indicativo de una escoria; en 1 b) se muestra el corte negro y poroso y la forma convexa de otra pieza procedente del mismo lugar.

En la figura 3 se presentan los difractogramas de las seis muestras. El correspondiente a la $\mathrm{n}^{\circ} 1$ presenta tres fases principales: calcita $\left(\mathrm{CaCO}_{3}\right)$, yeso $\left(\mathrm{CaSO}_{4} \cdot 2 \mathrm{H}_{2} \mathrm{O}\right)$ y FeO (wustita). También se observan picos correspondientes a magnetita $\left(\mathrm{Fe}_{3} \mathrm{O}_{4}\right.$, ) y goethita $(\mathrm{FeO}(\mathrm{OH}))$ y $\mathrm{SiO}_{2}$ (cuarzo). Los picos de $\mathrm{SiO}_{2}$ y de $\mathrm{Fe}_{3} \mathrm{O}_{4}$ no se han indicado en la figura para no complicarla más y son los siguientes: $\mathrm{SiO}_{2}$ picos principales a $2 \mathrm{O}=20,82^{\circ}$ y $26,65^{\circ} ; \mathrm{Fe}_{3} \mathrm{O}_{4}$, picos principales a $2 \Theta=30,13^{\circ} ; 35,48^{\circ} ; 43,1^{\circ} ; 57,0^{\circ}$ y $62,5^{\circ}$.

Pieza n 2: "Andelos" 85-86 Sc. Andueza Cata $11 \mathrm{c}$

Información arqueológica y descripción morfológica: Fondos de crisol, batería de hornos. Nuestra Sra. de Andión, Mendigorria (se cree del s. II a.C.)

Se plantea la posibilidad de que se trabajase a menor temperatura que en la del horno de Artajona $\left(\mathrm{n}^{\circ} 1\right)$, lo que significaría peor técnica que en aquélla.

No presenta magnetismo residual en pieza; en polvo algo, pero menos magnetismo que en la $\mathrm{n}^{\circ} 1$. Forma plano-convexa, aunque no conserva toda la forma; mucha porosidad, con poros grandes; presenta manchas de óxido ambiental. El color es menos oscuro que el de la $n^{\circ} 1$ de Artajona. Analizada la tierra adherida se comprueba que está constituida por carbonatos. Una vez limpia, su aspecto no cambia apreciablemente. Masa antes de la limpieza $=516,3 \mathrm{~g}$ y después $=176,9 \mathrm{~g}$. Densidad $=2,328 \mathrm{~g} / \mathrm{cm}^{3}($ resultado no muy fiable dada su elevada porosidad). El aspecto del corte, negro, es similar al que ofrece el de la pieza $n^{\circ} 1$ de Artajona. Estas observaciones 
acerca del parecido externo son pertinentes, porque como más adelante se concretará, también tienen composición análoga. El difractograma de rayos X muestra dos fases principales: wustita y calcita. También se observan picos de magnetita y algunos que pueden corresponder a goethita y cuarzo. El análisis por ICP no proporciona el total de elementos, pero por DRX se constata la presencia de carbono. $\mathrm{Al}$ igual que en la pieza $\mathrm{n}^{\circ} 1$ de Artajona, la excesiva concentración de hierro frente a silicio indica que no se formó fayalita, lo que se corrobora con la observación por microscopía óptica en la que no se ven cristales de la misma como es lo habitual, por lo que podríamos decir que sólo se llegaron a formar escorias del tipo clinopiroxeno que incluirían el calcio, cuyo porcentaje es relevante. De la fotografía no atacada a la atacada no se observan diferencias, en especial en las manchas blancas brillantes, que podrían ser de metal pero que no lo son al no verse afectadas por el ataque; son de wustita, forma en la que queda el hierro en exceso, si bien parte del porcentaje final de hierro podría provenir del retenido en la escoria dada su poca fluidez, como también ocurre en la pieza $\mathrm{n}^{\circ} 1$. Es de destacar que en esta muestra no aparecen las finas dendritas que se observaban después del ataque en la pieza $\mathrm{n}^{\circ} 1$. Alrededor de los alvéolos (manchas negras) se observan zonas blancas, más bien extensas, que se asemejan a las acumulaciones de cristales de magnetita. La presencia de magnetita fue constatada por DRX en polvo pero no hemos podido reconocer con seguridad estas mismas zonas en la observación por MEB por lo que la presunción de que sean de magnetita se ha hecho por comparación con las fotografías presentadas en la bibliografía ${ }^{[9]}$.

Esas manchas podrían ser de cuarzo e incluso de carbonato, que se pueden estudiar por MEB ya que están localizadas alrededor de alvéolos. La causa de que se forme magnetita se debe a no haberse completado la reducción, lo que indica falta de control en las condiciones de trabajo del horno tal como una ventilación excesiva.

En la figura 1 correspondiente al yacimiento $\mathrm{n}^{\mathrm{o}} 2$ "Escorias Andelos", en la fotografía 2 a) se muestra el aspecto general y tamaño, vista desde la cara plana. En 2 b) se observa la forma convexa y material adherido así como manchas de óxido.

En la figura 3, el difractograma correspondiente a la muestra $\mathrm{n}^{\circ} 2$ presenta dos fases principales wustita y calcita. También se observan picos de magnetita. Además presenta algunos picos que pueden corresponder a goethita y dos picos a $2 \Theta=26,6^{\circ}$ y $50,17^{\circ}$ de $\mathrm{SiO}_{2}$ (cuarzo).

Pieza no 3: Sartaguda Yac. 15 La Cerradilla Excavaciones de 1961. En otra ficha se señala, 4 ac $1 \mathrm{~s}, \mathrm{La}$ Cerradilla (romano).
Información arqueológica y descripción morfológica: Posibilidad de que contenga cobre. Magnetismo residual nulo. La forma es esférica, algo achatada; negra y con alvéolos pequeños. Podría haber ligeras irisaciones verdosas de cobre. La rodea mucha tierra salvo por un lado que podría ser una zona de corte, en esta zona su aspecto es muy vidriado y se mantiene después de limpiarla. La tierra que la rodea consiste en carbonato. Ya limpia, se ven claramente zonas con posibles irisaciones verdosas de cobre. También se observan los típicos surcos de haber fluido. Masa antes de limpiarla $=247,3 \mathrm{~g}$ y después $=241,3 \mathrm{~g}$. Densidad $=3,298 \mathrm{~g} / \mathrm{cm}^{3}$.

Ha resultado difícil de romper por su dureza. En el corte presenta tres capas que se aprecian en la foto y se describen a continuación: a) una zona superior más grisácea; b) una zona negra o muy oscura como las piezas de hierro de Arellano $\left(\mathrm{n}^{\circ} 6\right)$ y c) otra zona muy poco porosa y con cristalitos pequeños; en esta última se hallan intercaladas incrustaciones globulares de color marrón-ocre sin poros. Presenta dos fases principales: fatalita y cuarzo. La composición nos da una mayor proporción de $\mathrm{SiO}_{2}$ lo que permite formar una escoria más próxima a la ideal fayalítica.

Las fotografías muestran las agujas de fayalita perfectamente reconocibles, lo que supone enfriamiento lento en el interior del horno y sobre todo que se alcanzó la temperatura adecuada, en torno a los $1.100{ }^{\circ} \mathrm{C}$ para dar una escoria suficientemente fluida que permitiera arrastrar la ganga y retener menos metal. Se observan masas blanquecinas semejantes a las observadas en la pieza $n^{\circ} 2$ de Andelos y que fueron reconocidas como de magnetita aunque en este caso el análisis por DRX no permitió corroborar su presencia. Puesto que el análisis por DRX coincide con el análisis por ICP resultando ser el cuarzo una de las fases principales, parece que esas acumulaciones de cristales en este caso sí deberían ser de este compuesto, que provendría del utilizado como fundente y que quedó en exceso. Esto indicaría que no se controlaban las cantidades aportadas. El contenido en cobre de la muestra analizada mediante ICP es muy bajo y el análisis por DRX de polvo procedente de otras partes de la pieza no permitió un diagnóstico más preciso de su contenido, para así poder determinar si se podría tratar de una escoria de producción de cobre. Sin embargo en la observación al microscopio óptico se detectan manchas rojas y azules, tipo esmalte de mineral de cobre, por ello no se descarta que sean escorias de producción de cobre.

En la figura 1 correspondiente al yacimiento $\mathrm{n}^{\circ} 3$ "Sartaguda", la fotografía 3 a) muestra poros muy pequeños y la zona negra vidriada, $3 \mathrm{~b}$ ) muestra el aspecto general en forma de bola aplastada y el corte negro sin apenas poros. En 3 c) se observan las tres 
zonas en el corte de la pieza. Los nódulos de color marrón resultaron ser sílice.

En la figura 3, el difractograma correspondiente a la muestra $\mathrm{n}^{\mathrm{o}} 3$ presenta dos fases principales: cuarzo y fayalita.

Pieza $n^{\circ}$ 4: Areso escorias (tres piezas)

Información arqueológica y descripción morfológica: Son tres piezas (tortas de hierro) que no guardan la forma de un recipiente; su color es grisáceo y la base es más brillante con surcos, como prueba de haber fluido.

No hay alvéolos o en todo caso son muy pequeños. Presentan magnetismo residual cuando están en polvo. Apenas contienen tierra pegada, sólo pequeñas cantidades de carbonato en zonas puntuales. Masa antes de limpiarla $=142,8 \mathrm{~g}$ y después $=142,3 \mathrm{~g}$. Densidad $=2,278 \mathrm{~g} / \mathrm{cm}^{3}$.

Al partir la pieza por la zona central, se observan alvéolos grandes, con la pared granate. En conjunto, el corte presenta irisaciones agranatadas y signos de óxido. Presenta tres fases principales: fayalita, wustita y cuarzo.

$\mathrm{Al}$ igual que en la pieza $\mathrm{n}^{\circ} 3$ de Sartaguda, el análisis mediante ICP muestra una mayor proporción de $\mathrm{SiO}_{2}$, lo que permite formar una escoria más próxima a la ideal fayalítica. La observación con el microscopio óptico indica un fondo más homogéneo, propio de una escoria bien constituida; no obstante se observan muchos puntos blancos brillantes de $\mathrm{FeO}$ que indica presencia de hierro que no ha llegado a reducirse, lo cual está de acuerdo con la composición, pues para formar fayalita sólo se necesitaría aproximadamente $30 \%$ de $\mathrm{FeO}$. Lo más novedoso respecto al resto de muestras es la observación de manchas negras con un punto central que observadas por MEB resultaron ser poros (en forma de cono invertido) y su punto central era el fondo del poro del mismo material que el del resto de la escoria.

En la figura 1 correspondiente al yacimiento $\mathrm{n}^{\circ} 4$ "Areso escorias", en la fotografía 4 a) se aprecia el aspecto general de las tres piezas; $4 \mathrm{~b}$ ) muestra las irregularidades en la superficie pero no hay poros; también se pueden apreciar los surcos testigos de la existencia de deslizamiento.

En la figura 3, el difractograma correspondiente a la muestra $\mathrm{n}^{\circ} 4$ presenta tres fases principales: fayalita, wustita y cuarzo.

Pieza $n^{\circ}$ 5: Arellano, Alto cárcel

Información arqueológica y descripción morfológica: Se trata de una pieza de las mismas características que otras dos de hierro que provienen de una torta de un crisol de fundición de gran tamaño $80 \times 60 \times 60 \mathrm{~cm}$ (hueco de horno) y que pertenecen a época romana alrededor del siglo V. Según diversos autores tales como J. Chen ${ }^{[14]}$ se conocía el efecto de la adición de minerales de fósforo o bien fósforo de distinta procedencia para incrementar la dureza de las aleaciones férreas. Se precisa un análisis del contenido en fósforo. Ligero magnetismo residual en pieza. En piezas de $1 \mathrm{~cm}$ presenta magnetismo claro. Color herrumbroso, se diría que es claramente hierro; la base casi plana lleva tierra adherida (carbonatos) que una vez limpia deja al descubierto el mismo color herrumbroso y sigue siendo plana. El resto de la pieza tiene forma irregular, con fracturas y contornos de aristas marcadas. Masa antes de limpiarla $=359,9 \mathrm{~g}$ y después $=359 \mathrm{~g}$. Densidad $=4,053 \mathrm{~g} / \mathrm{cm}^{3}$

Resulta muy dura al tratar de trocearla. Presenta dos fases principales: goethita y magnetita. También se observan algunos picos de $\mathrm{SiO}_{2}$ y probablemente algo de $\mathrm{FeO}$. La composición muestra un alto porcentaje de $\mathrm{FeO}$, es decir de hierro que puede hallarse en diferentes combinaciones.

El bajo porcentaje de $\mathrm{SiO}_{2}$ ya indica que no estamos ante una escoria fayalítica como las anteriores, pero tampoco es hierro o mineral de hierro como lo demuestran las fotografías de la muestra pulida en las que encontramos hasta cuatro tipos de formaciones distintas que no incluyen agujas de fayalita. No obstante el aspecto general es muy homogéneo. Además de observarse escamas de magnetita identificadas mediante DRX, también aparece una zona de"granulado" blanco y negro que podría identificarse con la eutectoide perlita, lo que llevó a pensar en la presencia de carbono. El análisis mediante ICP no puede proporcionar el porcentaje de carbono que fue confirmado posteriormente mediante observación por MEB.

En la figura 1 correspondiente al yacimiento $n^{\circ} 5$ “Arellano, Alto Cárcel”, en la fotografía 5 a) se observa claramente la irregularidad de la pieza y las aristas vivas de algunos cortes; la $5 \mathrm{~b}$ ) muestra la zona de apoyo, más bien convexa.

En la figura 3, el difractograma correspondiente a la muestra $\mathrm{n}^{\circ} 5$ presenta dos fases principales: goethita y magnetita. También se observan los picos del cuarzo $\left(\mathrm{SiO}_{2}\right)$ : son los picos a $2 \Theta=26,47^{\circ}$ y $53,84^{\circ}$. Probablemente la muestra presente algo de $\mathrm{FeO}$, se observa el pico más intenso a $2 \Theta=42^{\circ}$.

Pieza ${ }^{\circ}$ 6: Arellano, (2 hierros)

Información arqueológica y descripción morfológica: Se trata de una pieza de las mismas características que otras dos de hierro que provienen, parece ser, de una torta de un crisol de fundición de gran tamaño 80x60x60 cm (hueco de horno) pertenecientes época romana alrededor del siglo V. Se trataría 
de una pieza que hay que relacionar con los hierros de Arellano ( $\left.\mathrm{n}^{\circ} 5\right)$ y por lo tanto valorar su contenido en fósforo que resulta ser inferior al de dicha pieza. Presenta magnetismo en fragmentos pequeños. Se aprecia en las fotografías que una de ellas tiene forma irregular, aunque próxima a cóncavo-convexa. Densidad: $3,856 \mathrm{~g} / \mathrm{cm}^{3}$.

Muy dura y corte negro, sin poros, parece tratarse de mineral. Esta muestra es muy similar a la muestra $\mathrm{n}^{\mathrm{O}} 5$. Presenta dos fases principales: goethita y magnetita. No presenta el pico de FeO. La composición refleja que prácticamente toda la muestra es $\mathrm{FeO}$, lo cual no entra en contradicción con la ausencia del pico de wustita, ya que mediante DRX se determinan fases cristalinas; esto quiere decir que si hay un compuesto amorfo o poco cristalino no se va a detectar su presencia mediante DRX, aunque el elemento hierro pueda ser detectado por técnicas de análisis químico como es la espectrometría ICP. La observación con el microscopio óptico indica asimismo una estructura homogénea y la presencia de manchas blancas antes del ataque que se observan negras después, por lo que se deduce que se trata de cristales de hierro metálico. La composición podría hacer pensar que se trata de mineral de hierro, pero la estructura indica que no. Al observar las fotografías de la pieza tal como se recibió, parece obvio que no se trata de un trozo de mineral arrancado de la roca a no ser que las altas temperaturas alcanzadas en el horno pudieran deformarlo y redondearlo, idea que apunta Gómez Ramos ${ }^{[5]}$. Podría tratarse de una escoria de alto contenido en FeO. Estadísticamente se pueden encontrar escorias con cualquier relación $\mathrm{FeO} / \mathrm{SiO}_{2}$ aun cuando estemos en épocas avanzadas ${ }^{[9]}$. Otra posibilidad es que se trate de una escoria obtenida en una herrería durante el proceso de forja. Ya se ha descrito la formación de una costra de $\mathrm{FeO}$ que se iría desprendiendo produciendo este tipo de desecho que no se originaría en horno de reducción. El análisis morfológico de la otra pieza recibida junto con ésta, permite avalar esta hipótesis. Aunque las piezas $\mathrm{n}^{\circ} 5 \mathrm{y} \mathrm{n}^{\circ} 6$ proceden de Arellano, pertenecen a dos lugares diferentes y dadas sus diferencias, podemos decir que en ambos se trabajaba de forma distinta.

En la figura 1 correspondiente al yacimiento $\mathrm{n}^{\circ} 6$ “Arellano, 2 hierros", la fotografia 6 a) muestra un aspecto muy homogéneo y de color herrumbroso; la 6 b) muestra otra pieza del mismo lugar de procedencia que la anterior.

En la figura 3, el difractograma correspondiente a la muestra $\mathrm{n}^{\circ} 6$ es muy similar al de la muestra $\mathrm{n}^{\circ} 5$. Presenta también dos fases principales: goethita y magnetita. En esta muestra no se observa el pico a $2 \mathrm{O}=42^{\circ}$ de $\mathrm{FeO}$.

\section{CONCLUSIONES}

- En primer lugar llama la atención que las piezas que tienen el mismo aspecto y características morfológicas parecidas han resultado tener también composición química similar, lo que ya permite una primera clasificación y una previsión de las pautas o líneas a seguir en su caracterización.

- La observación por microscopía de las piezas $\mathrm{n}^{\circ} 1$ $\mathrm{y}^{\mathrm{o}} 2$, de Artajona y Andelos respectivamente, indica la presencia de wustita. El mineral no ha llegado a reducirse por completo, y no presenta fayalita; el análisis por difracción lo confirma y completa con la identificación de carbonatos que formarían estructuras complejas tipo clinopiroxenos. Todo esto corresponde a escorias de estructura más bien rudimentaria ya que no se llegó a formar fayalita.

- En el caso de la pieza $n^{\circ} 2$, posible horno del siglo II a.C., el resultado estaría en consonancia con los obtenidos para época prerromana de la tesis de Gómez Ramos ${ }^{[5]}$ pero no en la $n^{\circ} 1$, si bien es más moderna ya que se observa la formación de dendritas; esto indica un mejor control de temperatura. Si realmente pertenece al siglo II d.C. estaríamos ante una técnica todavía rudimentaria para la época, a no ser que se tratara de una escoria, no de horno sino de forja del tipo SAS.

- La observación por microscopía de las piezas $\mathrm{n}^{\circ} 3$ y n $^{\circ} 4$, de Sartaguda y Areso, permite reconocer las agujas de fayalita así como formaciones de magnetita. Su estructura es más moderna, aunque lejos de la ideal que debería contener un $60 \%$ de wustita; el análisis por difracción aporta además la presencia de cuarzo que provendría del que ha quedado en exceso por falta de wustita y que indica falta de control sobre las cantidades a utilizar. La $\mathrm{n}^{\circ} 3$ pertenece a época romana, sin precisar siglo, lo que supone que la técnica era ya mejor. En el caso de la $n^{\circ} 4$ desconocemos los datos, aunque precisando algo más, podríamos compararla con las estudiadas por Kronz $z^{[9]}$ y deducir que se encontraría entre las S30 y S60 por lo que si son anteriores al s. IV d.C. serían buenas escorias para la época y si son posteriores o en torno al IV - V d.C. serían las propias de la época. Debido a la forma y los surcos superficiales se puede decir que proceden de un horno de fundición con sangrado, las porciones pastosas de escoria adquirirían la forma redondeada al rodar. Las piezas $\mathrm{n}^{\circ} 5 \mathrm{y} \mathrm{n}^{\circ} 6$, de distintos yacimientos de Arellano, son similares en aspecto y en composición química. Dado el 
alto contenido en hierro de ambas su origen es dudoso en el sentido de que no se trataría de escorias propiamente dichas. La observación mediante microscopía muestra un aspecto muy homogéneo con algunas variaciones singulares e incrustaciones que aportan información puntual pero no general acerca del tipo de pieza. El análisis por difracción da como fase relevante goethita por lo que como primera hipótesis se diría que son restos del mineral goethita, que usarían como mena, deformados por las altas temperaturas; esto vendría avalado por el hecho de que los difractogramas son muy parecidos, lo que indica que es el mismo tipo de pieza y que sólo puede tratarse de un mineral. No obstante, podemos establecer una segunda hipótesis, es decir que se trata de escorias de forja del tipo descrito como SFR, aunque nuestras piezas pertenecen a época romana del siglo $\mathrm{V}$ d.C. y estadísticamente las descritas como SFR son propias de época medieval. El análisis de las incrustaciones de la $\mathrm{n}^{\circ} 5$ indica que contiene carbono y es magnética. Para poder elegir entre las dos hipótesis deberíamos conocer el porcentaje de goethita en la muestra. En particular para la pieza $\mathrm{n}^{\circ} 5$ parece posible la utilización de fósforo para conseguir hierros de más dureza. En cualquier caso y esto es válido para todas las piezas, estamos hablando de una sola pieza por yacimiento por lo que deducir si en el lugar había un horno de fundición/reducción o un horno de herrería/forja y si la técnica era rudimentaria o acorde a la existente en el resto de España parece algo aventurado. Lo que sí se puede deducir es que usaban como mena goethita, ya que ésta aparece no sólo en las piezas $\mathrm{n}^{\mathrm{o}} 5 \mathrm{y} \mathrm{n}^{\mathrm{o}} 6$ sino también en las $\mathrm{n}^{\circ} 1$ y n $^{\circ} 2$.

- Finalmente podemos concluir que las técnicas que hemos utilizado en este trabajo han sido muy adecuadas para conseguir los objetivos propuestos de caracterización de las piezas y que se complementan entre sí.

\section{Agradecimientos}

Los autores desean expresar su agradecimiento a D. J. Sesma Sesma, Técnico Arqueólogo de la Sección de Arqueología del Gobierno de Navarra por su experta y amable colaboración.

\section{REFERENCIAS}

[1] R.F. Tylecote, A History of Metallurgy, The Institute of Metals, 1st. Ed., Londres, Inglaterra, 1976, pp. 64-80.

[2] P.T. Craddock y M.J. Hughes, Furnaces and Smelting Technology in Antiquity, British Museum (Occ. Papers 48), Londres, Inglaterra, 1985.

[3] P. Gómez -Ramos, Trabajos de prehistoria 1 (1996) 127-143.

[4] S. Rovira, Archaeometallurgy in Europe, Proceedings, Vol. 1, Associazione italiana di metallurgia, Milán, Italia, 2003, pp. 479-486.

[5] P. Gómez-Ramos, Tesis Doctoral, Facultad de Filosofía y Letras, Universidad Autónoma de Madrid, 1995.

[6] B. Rothenberg, Copper smelting furnaces in the Arabah, Israel: the archaeological evidence, Furnaces and Smelting Technology in Antiquity, P. Craddock y M. Hughes (Eds.), British Museum, Londres, Inglaterra, 1985, pp.123-150.

[7] A. Hauptmann, Archaeometallurgy in Europe, Proceedings, Vol. 1, Associazione italiana di metallurgia, Milán, Italia, 2003, pp. 459-468.

[8] J. Setién y J.A. Díez-Aja, Rev. Metal. 4 (2008) 299-309.

[9] A. Kronz, Archaeometallurgy in Europe, Proceedings, Vol. 1, Associazione italiana di metallurgia, 2003, pp. 555-564.

[10] H. Bachmann, Scientific Studies in Early Mining and Extractive Metallurgy, P. Craddock (Ed.), British Museum, Londres, Inglaterra, 1980, pp.103-134.

[11] V. Serneels y S. Perret, Archaeometallurgy in Europe, Proceedings, Vol. 1, Associazione italiana di metallurgia, Milán, Italia, 2003, pp. 469-478.

[12] J. Armendáriz, De aldeas a ciudades. El poblamiento durante el primer milenio a.C. en Navarra, Gobierno de Navarra, Trabajos de Arqueología Navarra, Monografías Arqueológicas, 2, 2008, pp. 77-80.

[13] G. Rosado y J.C. García, Rev. Metal. (Vol. Extr.) (2005) 101-106.

[14] J. Chen, M. Imamura y R. Han, Archaeometallurgy in Europe, Proceedings, Vol. 1, Associazione italiana di metallurgia, Milán, Italia, 2003, pp.77-86. 